\title{
4,4- bipiridin ve 2-amino-1,4-benzendikarboksilik asit tabanlı yeni bileşiğin sentezlenmesi ve hidrojen bağlı 3D polimerik yapısı
}

\author{
Görkem OYLUMLUOĞLU*
}

Fizik Bölümü, Moleküler Nano-Malzeme Labaratuvarı, Mugla Sitkı Kocman Üniversitesi, Mugla

Geliş Tarihi (Recived Date): 23.01.2018

Kabul Tarihi (Accepted Date): 06.03.2018

\begin{abstract}
Özet
Yeni bileşik, [ $\mathrm{NH}_{2}$-BDC][4,4-bpy], $\left(\mathrm{NH}_{2}\right.$-BDC = 2-amino-1,4-benzen dikarboksilik asit, 4,4-bipy = 4,4-bipiridin) sentezlendi ve tek kristal X-ışını kırınım metodu ile karakterize edildi. Kristalin yapısal analizi bileşiğin monoklinik uzay grubu I 2/a ile kristallendiği ve asimetrik biriminin iki yarım 2-amino-1,4-benzen dikarboksilik asit ve 4,4-bipiridin molekülü içerdiğini ve yapıda 1:1 oranında kristal oluşturduğunu göstermektedir. $\mathrm{NH}_{2}$ BDC molekülü simetri merkezi üzerinde yer alırken 4,4-bipy molekülü iki katlı dönme ekseninde bulunmaktadır. Kristal yapıda, moleküller ü̧̈ adet kuvvetli molekül içi ve moleküler arası $O-H \cdots N$ ve $O-H \cdots O$ hidrojen bağları ile bir arada tutunurlar ve $3 D$ kristal yapısını oluşturur. Hidrojen bağlı polimerik ăg bc düzleminde paketlenir ve a ekseni boyunca istiflenir. Buna ek olarak, kristal $\mathrm{C}-H \cdots O$ tip zaylf hidrojen bağl ile daha kararlı hal alır. Kristal paketlenmesinde aromatik halkaların bir merkezden diğer merkeze 3.740(2) А uzaklı̆̆a sahip $\pi-\pi$ etkileşmelerinin olduğu görülür. Sarı renkli

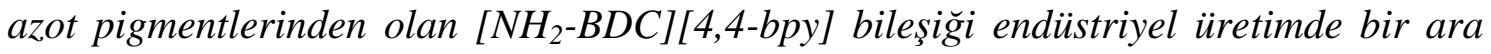
madde olarak kullanılabilir.
\end{abstract}

Anahtar Kelimeler: 2-amino-1 4-benzene dicarboxylic acid, 4,4-bipyridine, kristal yapl.

\footnotetext{
* Görkem OYLUMLUOĞLU, gorkem@ @u.edu.tr, http://orcid.org/0000-0002-7398-4018
} 


\title{
Synthesis and hydrogen bonded 3D polymeric structure of new compound based on 2-amino-1,4-benzene dicarboxylic acid and 4,4-bipyridine
}

\begin{abstract}
The new compound, [NH $\left.\mathrm{H}_{2}-\mathrm{BDC}\right]\left[4,4\right.$-bpy], $\left(\mathrm{NH}_{2}-\mathrm{BDC}=2\right.$-amino-1,4-benzene dicarboxylic acid, 4,4-bipy = 4,4-bipyridine) was synthesized and characterized by single crystal $X$-ray diffraction method. The crystal structure analysis indicates that complex crystallizes in the monoclinic space group I 2/aand its asymmetric unit of contains two half2-amino-1,4-benzene dicarboxylic acid and 4,4-bipyridine molecules, which constitute a 1:1 co-crystal. The $\mathrm{NH}_{2}-\mathrm{BDC}$ molecule is situated on an inversion center while the 4,4-bipy molecule is situated on a twofold rotational axis. In the crystal structure, molecules are held together via three strong intramolecular and intermolecular $O-H \cdots N$ and $O-H \cdots O$ hydrogen bonds, resulting in $3 D$ structure. This hydrogen-bonded polymeric network lies in the bc plane and stacks along the a axis. Furthermore, the crystal is stabilized by weak hydrogen bond of the $\mathrm{C}-\mathrm{H} \cdots \mathrm{O}$ type. The crystal packing exhibits $\pi-\pi$ interactions between the aromatic rings with a centroidcentroid distance of 3.740(2) $\AA$. The compound, [NH $-\mathrm{BDC}][4,4-$ bpy], which is a yellow azo pigments can be used as an intermediate in industrial production.
\end{abstract}

Keywords: 2-amino-1,4-benzene dicarboxylic acid, 4,4-bipyridine, crystal structure.

\section{Giriş}

Son yıllarda, karboksilik asitler gözenekli metal organik çerçeve yapılı bileşiklerin, senteziyle ilgili olarak büyük ilgi görmüştür. Organik bağlayıcı molekülünün çözücü içindeki davranış bilgisi, asidik karakter ve dolayısıyla sentez stratejisi hakkında önemli bilgiler sağlayabilir [1]. Sarı Pigment gibi sanayide kullanılan azot pigmentlerinin sentezinde 2-amino-1,4-benzene dicarboxylic acid sıklıkla kullanılmaktadır. Bu bileşikler, 1912 yılından bu yana bilinmektedir ve antitümör ilaçlarda reflü hastalığına karşı ilaçlarda pigmentlerin sentezlenmesinde polimerlerin hazırlanmasında ve parallel sentezlemelerde kullanıldı ̆̆ gibi birçok araştırmaya da konu olmuştur [2]. Bununla birlikte bipiridin türevleri de polimerik koordinasyon bileşiklerinde genellikle bir bağlayıcı olarak yaygın kullanılan bir moleküldür [3]. Fonksiyonel Gruplarla birlikte birçok aromatik türün, kristal mühendisliği için uygun tektonlar sağlayan özel moleküler etkileşimler geliştirdiği bilinmektedir. Özellikle, aromatik karboksilik asit ve amino grupları, hidrojen bağı verici ve alıcıları olarak görev yapabilir ve hidrojen bağlarının yönünü yaklaşık moleküler düzlemde uzun tutabilir. Genellikle, bu tür hidrojen bağı etkileşimlerinin uzantısı, bir boyutlu makro moleküllere öncülük etmektedir. Kristal paketlenmesi etkileşimleri ele alındığında, bantlar veya iki boyutlu makro molekül katmanları üç boyutlu bir iskelete bağlanır. Fonksiyonel Organik Çerçeveler katıların tasarımında ve spesifik özelliklere sahip metal organik çerçeve yapılarında yaygın olarak kullanılır. 2-Aminobenzen-1,4-dikarboksilik asit, sistematik yapısal tasarımda metan depolamasında olası uygulamalarla birlikte metal organik çerçeveler için bir ligand olarak iyi bilinir $[4,5]$. 
Yakın zamanda, araştırma grubumuz çeşitli aromatik amid-karboksilatlar içeren küçük organik moleküllerde hidrojen bağ etkileşimlerini çalışmıştır [6-8]. Yukarıda bahsedilen ilgi alanları ışığında ve daha önceki çalışmalarımızın devamı olarak yeni [ $\mathrm{NH}_{2}$-BDC][4,4-bpy], $\left(\mathrm{NH}_{2}\right.$-BDC = 2-amino-1,4-benzen dikarboksilik asit, 4,4-bipy = 4,4-bipiridin) bileșiği sentezlendi ve tek kristal X-ışını kırınım metodu ile karakterize edildi. Ayrıca kristal yapıdaki molekül içi ve moleküler arası $\mathrm{O}-\mathrm{H} \cdots \mathrm{N}, \mathrm{O}-\mathrm{H} \cdots \mathrm{O}, \mathrm{C}-$ $\mathrm{H} \cdots \mathrm{O}$ hidrojen bağları ve $\pi-\pi$ etkileşimleri incelendi.

\section{Materyal ve metot}

Deneyde kullanılan kimyasal maddeler Merck, Fluka, ve Sigma-Aldrich firmalarından ticari olarak alınmıștır ve herhangi bir saflaștırma ișlemi uygulamadan kullanılmıștır. Kompleksin element analizi LECO marka cihaz ve Tek kristal X-1şınları analizi Oxford Diffraction Xcalibur-3 X-Işınları Tek Kristal Difraktometre cihazı ile Mo-Ka 1şını kullanılarak oda sıcaklığında ölçülmüştür [9]. Bileşiğin kristal yapısı, direk yöntem kullanılarak SHELXS programı ile OLEX2 de çözüldü $[10,11]$. Hidrojen dışındaki bütün atomlar anizotropik yer değiştirme parametresi olarak tanımlandı ve konumsal kısıtlama olmaksızın revize edildi. Hidrojen atomları ideal bir şekilde konumlandırılarak izotropik yer değiştirme parametresi kendilerine bağlı olan karbon atomlarının $U_{\text {equiv }}$ nın 1,2 katına sınırlandırıldı. Moleküller arası $\pi$ etkileşimleri ise detaylı bir şekilde PLATON 1.17 programı ile hesaplandı [12].

\subsection{Bileşiğin sentezi}

2-amino-1,4-benzen dikarboksilik asit $(1 \mathrm{mmol})$ ve 4,4'- bipiridin (1 mmol), $20 \mathrm{ml}$ $\mathrm{H}_{2} \mathrm{O} / \mathrm{DMF}$ çözeltisinde kaynama noktasına kadar isıtılarak çözülmüş ve bu çözeltiye 5 ml NaOH eklenerek PH: 4.11 değerine yükseltilerek, çözelti 20 dakika daha manyetik karıştırıcı ile karıştırılmıştır. Elde edilen çözelti daha sonra teflon kaplı bir otoklava (basınçlı kap) aktarılmıştır. Reaktör, oda sıcaklığından $120{ }^{\circ} \mathrm{C}$ dereceye kadar 12 saatte 1sitılıp, 50 saat $120{ }^{\circ} \mathrm{C}$ sicaklıkta tutulup ve 24 saatte oda sicaklığına düşecek şekilde programlanan etüve yerleştirilmiştir. Çözelti yaklaşık iki hafta boyunca oda sıcaklığında bekletilmiş ve sarı renkli tek kristaller süzme kâğıdıyla ayrıştırılmıştır.

Verim \% 80. Teorik element analizi (\%) $\left(\mathrm{C}_{8} \mathrm{H}_{8} \mathrm{~N}_{2} \mathrm{O}_{4}\right)\left(\mathrm{C}_{10} \mathrm{H}_{8} \mathrm{~N}_{2}\right): \mathrm{C} 61.36, \mathrm{H} 4.58, \mathrm{~N}$ 15.9; Deneysel element analizi (\%): C 61.21, H 4.57, N 15.8.

\section{Bulgular}

\subsection{Kristal yapı analizi}

[ $\mathrm{NH}_{2}$-BDC][4,4-bpy] bileșiğine ait kristalografik bilgiler Tablo 1'de, bağ uzunlukları ve bağ açıları ise Tablo 2'de, Hidrojen bağları ve $\pi-\pi$ etkileşimleri ise Tablo 3 ' de verilmektedir. Bileşiğin moleküler yapısının görünümü Şekil 1'de, molekül içi ve moleküller arası O-H $\cdots \mathrm{N}, \mathrm{O}-\mathrm{H} \cdots \mathrm{O}$ hidrojen bağlarının görünümü Şekil 2'de, üç boyutta paketlenmiş görünümü Şekil 3'de ve kristal yapıdaki aromatik halkalar arasındaki $\pi-\pi$ etkileşimleri ise Şekil 4 de verilmektedir. 


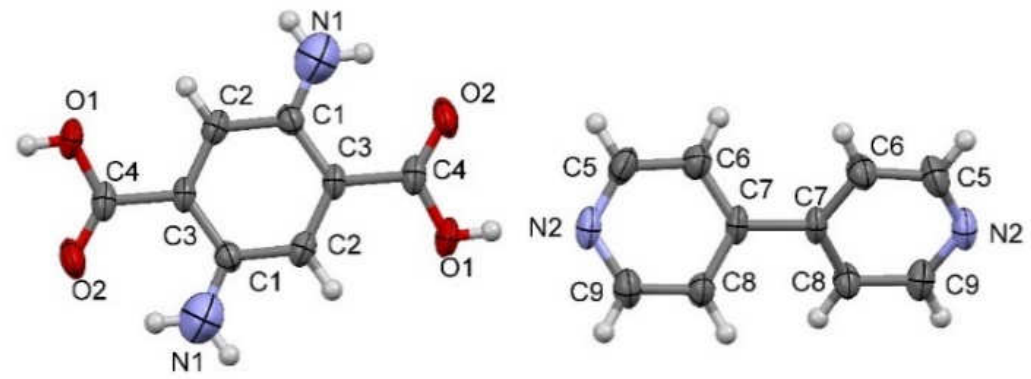

Şekil 1. Bileşiğin moleküler yapısı.

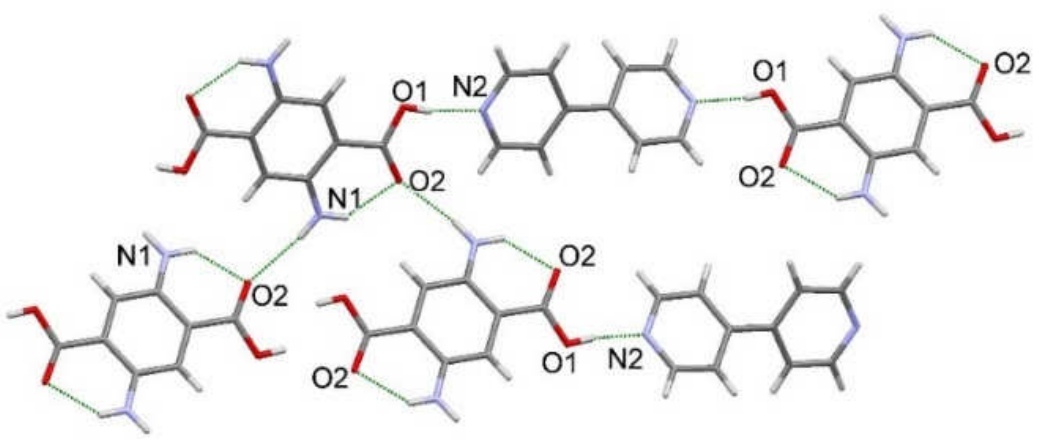

Şekil 2. Bileşikteki molekül içi ve moleküller arası $\mathrm{O}-\mathrm{H} \cdots \mathrm{N}$ ve $\mathrm{O}-\mathrm{H} \cdots \mathrm{O}$ hidrojen bağlarının görünümü.
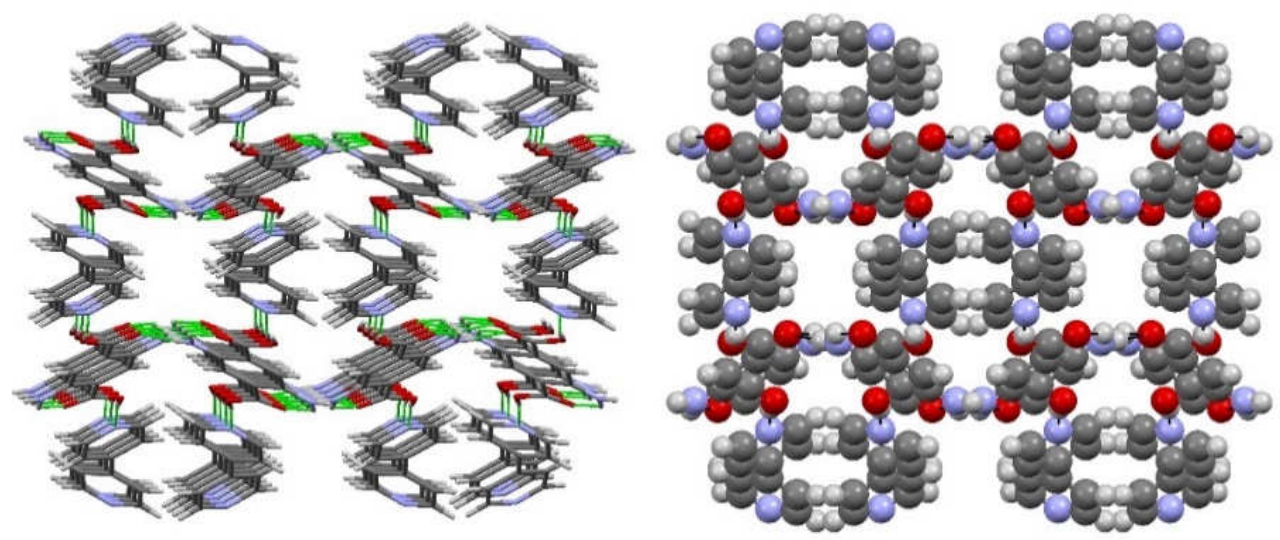

Şekil 3. Bileşikteki moleküllerin $b c$ ekseninde üç boyutta (3D) paketlenmiş görünümü.

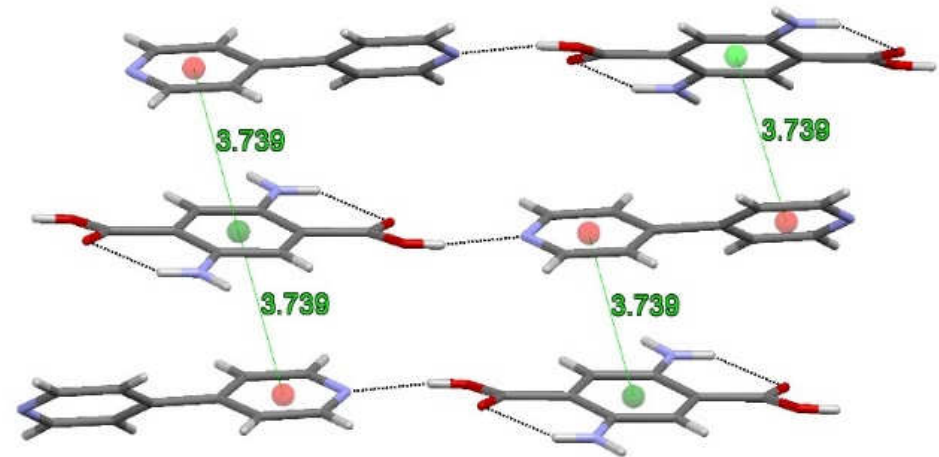

Şekil 4. Kristal yapıdaki $\pi-\pi$ etkileşmesinin görünüşü. 
Tablo 1. Moleküle ait kristalografik bilgiler ve deneysel veriler.

\begin{tabular}{ll}
\hline CCDC No & 1817340 \\
Molekülün Kapalı Formülü & $\mathrm{C}_{8} \mathrm{H}_{8} \mathrm{~N}_{2} \mathrm{O}_{4} \mathrm{C}_{10} \mathrm{H}_{8} \mathrm{~N}_{2}$ \\
Molekülün Ağırlığı $\left(\mathrm{g} \mathrm{mol}^{-1}\right)$ & 352.35 \\
Kristal Sistemi, Uzay Grubu & Monoklinik, I2/a \\
Sıcaklık $(\mathrm{K})$ & 293 \\
Birim Hücre Parametreleri $a, b, c(\AA)$ & $9.2735(9), 11.2218(16), 15.2777(17)$ \\
$\beta\left({ }^{\circ}\right)$ & $95.842(10)$ \\
Birim Hücre Hacmi $\left(\AA^{3}\right)$ & $1581.6(3)$ \\
Birim Hücredeki Molekül Sayısı & 4 \\
$\mu\left(\mathrm{mm}^{-1}\right)$ & 0.11 \\
Ölçülen Yansıma & 2623 \\
Bağımsı Yansıma & 1608 \\
Gözlenen Yansıma Sayılları & $1156[I>2 \sigma(I)]$ \\
$R_{\text {int }}$ & 0.014 \\
$R\left[F^{2}>2 \sigma\left(F^{2}\right)\right], w R\left(F^{2}\right), S$ & $0.095,0.303,1.12$ \\
İncelenen Parametre Sayısı & 1608 \\
$\Delta \rho_{\max }, \Delta \rho_{\min }\left(\mathrm{e} \AA^{-3}\right)$ & $0.41,-0.63$ \\
\hline
\end{tabular}

Tablo 2. Seçilen geometrik parametreler $\left(\AA{ }^{\circ}{ }^{\circ}\right)$.

\begin{tabular}{|c|c|c|c|}
\hline $\mathrm{O} 2-\mathrm{C} 4$ & $1.207(5)$ & $\mathrm{C} 7-\mathrm{C}^{\mathrm{ii}}$ & $1.482(6)$ \\
\hline $\mathrm{O} 1-\mathrm{C} 4$ & $1.311(5)$ & $\mathrm{C} 7-\mathrm{C} 8$ & $1.389(5)$ \\
\hline $\mathrm{N} 2-\mathrm{C} 9$ & $1.332(6)$ & $\mathrm{C} 7-\mathrm{C} 6$ & $1.379(5)$ \\
\hline $\mathrm{N} 2-\mathrm{C} 5$ & $1.320(6)$ & $\mathrm{C} 1-\mathrm{C} 2$ & $1.388(5)$ \\
\hline $\mathrm{C} 3-\mathrm{C} 4$ & $1.487(5)$ & $\mathrm{C} 1-\mathrm{N} 1$ & $1.255(8)$ \\
\hline $\mathrm{C} 3-\mathrm{Cl}^{\mathrm{i}}$ & $1.403(5)$ & $\mathrm{C} 8-\mathrm{C} 9$ & $1.379(5)$ \\
\hline $\mathrm{C} 3-\mathrm{C} 2$ & $1.380(5)$ & $\mathrm{C} 6-\mathrm{C} 5$ & $1.383(6)$ \\
\hline $\mathrm{C} 5-\mathrm{N} 2-\mathrm{C} 9$ & $117.6(3)$ & $\mathrm{O} 1-\mathrm{C} 4-\mathrm{C} 3$ & $113.4(4)$ \\
\hline $\mathrm{Cl}^{\mathrm{i}}-\mathrm{C} 3-\mathrm{C} 4$ & $120.2(3)$ & $\mathrm{C} 2-\mathrm{C} 1-\mathrm{C} 3^{\mathrm{i}}$ & $119.3(4)$ \\
\hline $\mathrm{C} 2-\mathrm{C} 3-\mathrm{C} 4$ & $120.4(3)$ & $\mathrm{N} 1-\mathrm{C} 1-\mathrm{C} 3^{\mathrm{i}}$ & $121.0(4)$ \\
\hline $\mathrm{C} 2-\mathrm{C} 3-\mathrm{C}^{\mathrm{i}}$ & $119.4(3)$ & $\mathrm{N} 1-\mathrm{C} 1-\mathrm{C} 2$ & $119.7(4)$ \\
\hline $\mathrm{C} 8-\mathrm{C} 7-\mathrm{C}^{\mathrm{ii}}$ & $120.1(2)$ & $\mathrm{C} 3-\mathrm{C} 2-\mathrm{C} 1$ & $121.3(3)$ \\
\hline $\mathrm{C} 6-\mathrm{C} 7-\mathrm{C} 7^{\mathrm{ii}}$ & $122.3(3)$ & $\mathrm{C} 9-\mathrm{C} 8-\mathrm{C} 7$ & $119.0(4)$ \\
\hline $\mathrm{C} 6-\mathrm{C} 7-\mathrm{C} 8$ & $117.6(3)$ & $\mathrm{N} 2-\mathrm{C} 9-\mathrm{C} 8$ & $123.2(4)$ \\
\hline $\mathrm{O} 2-\mathrm{C} 4-\mathrm{O} 1$ & $123.3(3)$ & $\mathrm{C} 7-\mathrm{C} 6-\mathrm{C} 5$ & $119.3(4)$ \\
\hline $\mathrm{O} 2-\mathrm{C} 4-\mathrm{C} 3$ & $123.3(4)$ & $\mathrm{N} 2-\mathrm{C} 5-\mathrm{C} 6$ & $123.3(4)$ \\
\hline $\mathrm{C} 3{ }^{\mathrm{i}}-\mathrm{C} 1-\mathrm{C} 2-\mathrm{C} 3$ & $-0.5(6)$ & $\mathrm{C} 1^{\mathrm{i}}-\mathrm{C} 3-\mathrm{C} 2-\mathrm{C} 1$ & $0.5(6)$ \\
\hline $\mathrm{C} 7^{\mathrm{ii}}-\mathrm{C} 7-\mathrm{C} 8-\mathrm{C} 9$ & $-179.1(4)$ & $\mathrm{C} 2-\mathrm{C} 3-\mathrm{C} 4-\mathrm{O} 2$ & $-170.5(4)$ \\
\hline $\mathrm{C} 7^{\mathrm{ii}}-\mathrm{C} 7-\mathrm{C} 6-\mathrm{C} 5$ & $178.2(4)$ & $\mathrm{C} 2-\mathrm{C} 3-\mathrm{C} 4-\mathrm{O} 1$ & $10.8(5)$ \\
\hline $\mathrm{C} 7-\mathrm{C} 8-\mathrm{C} 9-\mathrm{N} 2$ & $0.9(6)$ & $\mathrm{C} 8-\mathrm{C} 7-\mathrm{C} 6-\mathrm{C} 5$ & $-0.4(6)$ \\
\hline $\mathrm{C} 7-\mathrm{C} 6-\mathrm{C} 5-\mathrm{N} 2$ & $0.9(7)$ & $\mathrm{C} 9-\mathrm{N} 2-\mathrm{C} 5-\mathrm{C} 6$ & $-0.5(7)$ \\
\hline $\mathrm{C} 4-\mathrm{C} 3-\mathrm{C} 2-\mathrm{C} 1$ & $-179.6(3)$ & $\mathrm{C} 6-\mathrm{C} 7-\mathrm{C} 8-\mathrm{C} 9$ & $-0.4(6)$ \\
\hline $\mathrm{C} 1^{\mathrm{i}}-\mathrm{C} 3-\mathrm{C} 4-\mathrm{O} 2$ & $9.4(6)$ & $\mathrm{C} 5-\mathrm{N} 2-\mathrm{C} 9-\mathrm{C} 8$ & $-0.4(7)$ \\
\hline $\mathrm{C}_{1}^{\mathrm{i}}-\mathrm{C} 3-\mathrm{C} 4-\mathrm{O} 1$ & $-169.3(4)$ & $\mathrm{N} 1-\mathrm{C} 1-\mathrm{C} 2-\mathrm{C} 3$ & $-178.7(5)$ \\
\hline
\end{tabular}


Bileşik monoklinik kristal sistemi $\mathrm{I} 2 / \mathrm{a}$ uzay grubunda kristalleşmiştir. Bileşiğin asimetrik birimi, 1: 1 oranında iki yarım 2-amino-1,4-benzen dikarboksilik asit ve 4,4bipiridin moleküllerini içerir. 2-amino-1,4-benzen dikarboksilik asit molekülü simetri merkezinde yer almaktadır. Karboksil gruplarının merkezi altı elemanlı halkası düzlemseldir [en iyi düzlemden ortalama sapma: 0.001 (1) $\AA$ ] ve karboksil grupları, benzen halka düzlemi arasındaki dihedral açı 10.71 (1) ${ }^{\circ}$ dir. 4,4'-bipiridin molekülü iki katlı dönme ekseni üzerinde bulunur ve iki piridin halkası 38.1 (1) ${ }^{\circ}$ lik bir dihedral açı oluşturur. Tüm bağ uzunlukları ve bağ açıları benzer daha önceden rapor edilen bileşiklerle iyi bir uyum içindedir (Tablo 2) [1-6]. Kristal yapıda, moleküller 3 adet güçlü molekül içi ve moleküler arası $\mathrm{O}-\mathrm{H} \cdots \mathrm{N}, \mathrm{O}-\mathrm{H} \cdots \mathrm{O}$ hidrojen bağları ile bir arada tutulur (Şekil 2). Bu hidrojen-bağlı polimerik ağ $b c$ düzleminde bulunur ve $a$ eksen boyunca istiflenerek üç boyutlu yapıyı (3D) oluşturur (Şekil 3). Ayrıca, kristal CH...O zayıf hidrojen bağı ile daha kararlı hal alır. Kristal yapı paketlenmesinde, aromatik halkaların merkezleri arasında 3.740 (2) A' luk merkez-merkez mesafesi ile $\pi$ $\pi$ etkileşimleri de gözlenmektedir (Şekil 4).

Tablo 3. Hidrojen bağları $\left(\AA{ }^{\circ}\right), \mathrm{Cg}-\mathrm{Cg}$ mesafeleri ile kısa halka etkileşmeleri.

\begin{tabular}{|c|c|c|c|c|c|}
\hline D-H....A* & D-H & H...A & D...A & D-H...A & Symmetry Code \\
\hline $\mathrm{O} 1-\mathrm{H} 1 \ldots \mathrm{N} 2$ & 0.82 & 1.84 & $2.648(4)$ & 168 & \\
\hline N1-H1A...O2 & 0.86 & 2.12 & $2.972(7)$ & 171 & $1-\mathrm{x}, 1 / 2+\mathrm{y}, 3 / 2-\mathrm{z}$ \\
\hline N1-H1B...O2 & 0.86 & 1.94 & $2.618(7)$ & 135 & $3 / 2-x, 1 / 2-y, 3 / 2-z$ \\
\hline $\mathrm{C} 2-\mathrm{H} 2 \ldots \mathrm{O} 1$ & 0.93 & 2.39 & $2.715(5)$ & 100 & \\
\hline \multicolumn{6}{|l|}{$\mathbf{C g}(\mathbf{I}) \ldots \mathrm{Cg}(\mathrm{J})$} \\
\hline $\mathrm{Cg}(1) \ldots \mathrm{Cg}(2)$ & & & $3.739(2)$ & & $1+\mathrm{x}, \mathrm{y}, \mathrm{z}$ \\
\hline $\operatorname{Cg}(1) \ldots \operatorname{Cg}(2)$ & & & $3.739(2)$ & & $1 / 2-x, 1 / 2-y, 3 / 2-z$ \\
\hline $\operatorname{Cg}(2) \ldots \operatorname{Cg}(1)$ & & & $3.739(2)$ & & $-1+\mathrm{x}, \mathrm{y}, \mathrm{z}$ \\
\hline $\operatorname{Cg}(2) \ldots \operatorname{Cg}(1)$ & & & $3.739(2)$ & & $1 / 2-x, 1 / 2-y, 3 / 2-z$ \\
\hline
\end{tabular}

D: Verici, A: Alıcı, Cg(I): Düzlem numarası I (halka numarası () içinde yukarıda),

$\mathrm{Cg}(1)$ : C2-C3-C3-C1a-C2a-C3a, Cg(2): N2-C5-C6-C7-C8-C9

\section{Tartışma ve sonuç}

Hidrotermal yöntem kullanılarak, [NH2-BDC][4,4-bpy], (NH2-BDC = 2-amino-1,4benzen dikarboksilik asit, 4,4-bipy =4,4-bipiridin) bileşiği sentezlenmiştir. Sentezlenen yeni bileşik, Tek kristal X-ışını kırınımı tekniği ile yapısal olarak karakterize edilmiştir. Kristal paketlenmesinde aromatik halkaların bir merkezden diğer merkeze $\pi-\pi$ etkileşmelerinin olduğu görülmüştür. Sarı renkli azot pigmentlerinden olan [NH2BDC][4,4-bpy] bileşiği endüstriyel üretimde ve sanayide bir ara madde olarak kullanılabilmektedir.

\section{Ek veriler}

$\mathrm{Bu}$ makalede verilen kristalografik veriler, Cambridge Crystallographic Data Centre'da depolanmış olup talep üzerine, (The Director, CCDC, 12 Union Road, Cambridge, CB2 1EZ, UK; e-mail: deposit@ccdc.cam.uk; www: http://www.ccdc.cam.ac.uk; fax: +44 1223 336033) CCDC 1817340 No.lu depolama numarası ile ücretsiz olarak temin edilebilir. 


\section{Teșekkür}

Tek Kristal X-1şınları ölçümleri için Dokuz Eylül Üniversitesi Fen Fakültesi Fizik bölümüne ve Yrd. Doç. Dr. Muhittin AYGÜN'e teşekkür ederim.

\section{Kaynaklar}

[1] Loos, S., Seichter, W., Weber, E. ve Mertens, F., 2-Aminoterephthalic acid N,N Dimethylformamide disolvate, Acta Crystallographica Section E, E68, 25012508, (2012).

[2] Brüning, J., Bats, J.W. ve Schmidt, M.U., 2-Aminoterephthalic acid dimethyl ester, Acta Crystallographica Section E, E65, 2468-2469, (2009).

[3] Zhang, W.X., Liao, P.Q., Lin, R.B., Wei, Y.S., Zeng, M.H. ve Chen, X.M., Metal cluster-based functional porous coordination polymers, Coordination Chemistry Reviews, 293-294, 263-268, (2015).

[4] Yabing, H., Wei, Z., Guodong, Q. ve Banglin, C., Methane storage in metalorganic frameworks, Chemical Society Reviews, 43, 5657, (2014).

[5] Qiu, S.L. ve Zhu, G.S., Molecular engineering for synthesizing novel structures of metal-organic frameworks with multifunctional properties, Coordination Chemistry Reviews, 253, 2891-2911, (2009).

[6] Sen, I., Kara, H. ve Azizoglu, A., Substituent effects on hydrogen bonding of aromatic amide-carboxylate, Spectrochimica Acta Part A: Molecular and Biomolecular Spectroscopy, 167, 50-58, (2016).

[7] Coban, M.B., Kocak, C., Kara, H., Aygun, M. ve Amjad, A., Magnetic properties and sensitized visible and NIR luminescence of Dy-III and Eu-III coordination polymers by energy transfer antenna ligands, Molecular Crystals and Liquid Crystals, 648, 202-215, (2017).

[8] Coban, M.B., Amjad, A., Kara, H. ve Aygun, M., Sensitization of Ho-III and Sm-III luminescence by efficient energy transfer from antenna ligands: Magnetic, visible and NIR photoluminescence properties of Gd-III, Ho-III and Sm-III coordination polymers, Inorganica Chimica Acta, 455, 25-33, (2017).

[9] SMART, Copyright 1989-1999, Bruker AXS, Madison, WI, USA; SAINT, Bruker AXS, Madison, WI, USA.

[10] Sheldrick, G.M., A short history of SHELX, Acta Crystallographica Section A Foundations and Advances, 64, 112-122, (2008).

[11] Dolomanov, O.V., Bourhis, L.J., Gildea, R.J., Howard, J.A.K. ve Puschmann, H., OLEX2: A complete structure solution, refinement and analysis program, Journal of Applied Crystallography, 42, 339-341, (2009).

[12] Spek, A.L., Structure validation in chemical crystallography, Acta Crystallographica Section D Biological Crystallography, 65,148-155, (2009). 